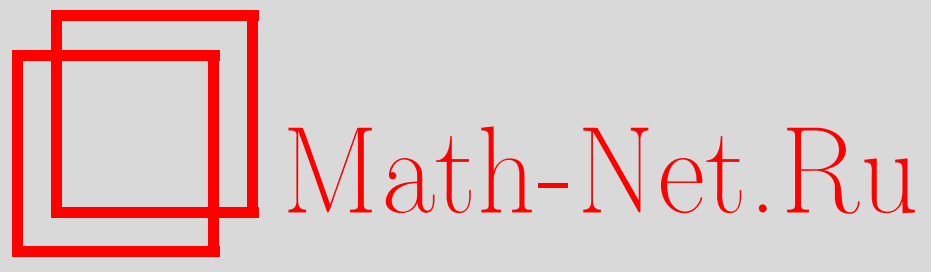

П. Константин, Локальные формулы для гидродинамического давления и их приложения, УМH, 2014, том 69, выпуск 3, 3-26

DOI: https://doi.org/10.4213/rm9590

Использование Общероссийского математического портала Math-Net.Ru подразумевает, что вы прочитали и согласны с пользовательским соглашением http://www . mathnet.ru/rus/agreement

Параметры загрузки:

IP : 54.205 .225 .156

26 апреля 2023 г., 12:28:18

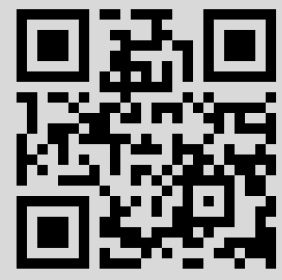




\section{Локальные формулы для гидродинамического давления и их приложения}

\section{П. Константин}

Предложены локальные формулы для давления в несжимаемой жидкости. Давление выражается через среднее давление и средние значения квадратов приращений скорости в произвольно малой окрестности. В качестве приложения приводится короткое доказательство того факта, что для скоростей класса гладкости $C^{\alpha}$ давление имеет гладкость $C^{2 \alpha}$ (или непрерывно по Липшицу). Также приводятся критерии регулярности для уравнений Навье-Стокса 3-мерной несжимаемой жидкости.

Библиография: 9 названий.

Ключевые слова: уравнения Навье-Стокса, уравнения Эйлера, давление, критерии регулярности.

DOI: $10.4213 / \mathrm{rm} 9590$

\section{СоДЕРЖАНИЕ}

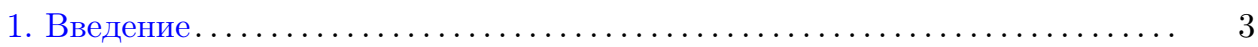

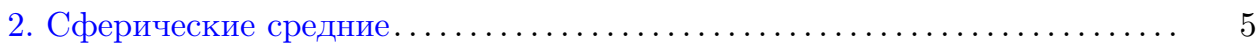

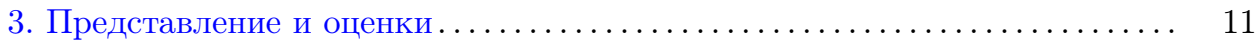

4. FGТ-оценки [4] во всем пространстве ....................... 15

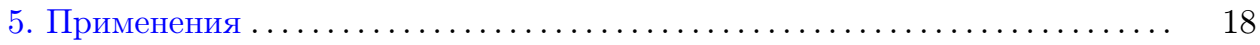

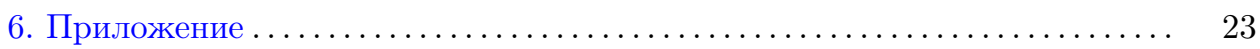

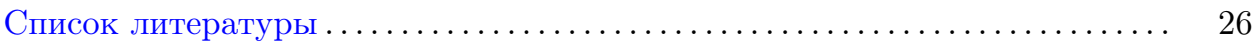

\section{1. Введение}

Мы предлагаем локальные формулы для давления в несжимаемой жидкости. А именно, имеются в виду выражения для вычисления в точке $x \in \Omega \subset \mathbb{R}^{3}$ значения решения уравнения

$$
-\Delta p=\sum_{i, j=1}^{3} \frac{\partial^{2}}{\partial x_{i} \partial x_{j}}\left(u_{i} u_{j}\right)
$$

Работа выполнена при поддержке грантами NSF-DMS 1209394 и NSF-DMS 1265132.

(C) П. Константин, 2014 
где $u$ - бездивергентное поле скоростей, через сферические средние давления

$$
\bar{p}(x, r)=\frac{1}{4 \pi r^{2}} \int_{|x-y|=r} p(y) d S(y)
$$

и интегралы от приращений $\left(u_{i}(y)-u_{i}(x)\right)\left(u_{j}(y)-u_{j}(x)\right)$ по шару $|y-x| \leqslant r$, где $r$ сколь угодно мало. Нам не требуется информация о поведении $u$ вне маленького шара. Основной момент доказательства - уравнение типа монотонности для модифицированной величины

$$
b(x, r)=\bar{p}(x, r)+\frac{1}{4 \pi r^{2}} \int_{|x-y|=r}\left(\frac{y-x}{|y-x|} \cdot u(y)\right)^{2} d S(y) .
$$

С его помощью давление выражается в виде

$$
p(x)=\beta(x, r)+\pi(x, r),
$$

где $\beta$ - просто среднее от давления по "окрестности":

$$
\beta(x, r)=\frac{1}{r} \int_{r}^{2 r} \bar{p}(x, \rho) d \rho,
$$

а $\pi(x, r)$ задается парой интегралов (39) от квадратов приращений скорости, по шару и по шаровому кольцу радиуса $2 r$. Таким образом, давление представляется в виде суммы двух локальных членов, один из которых мал, а второй имеет регулярное поведение. В самом деле, $\beta \in L^{\infty}\left(\mathbb{R}^{3}\right)$ - ограниченная функция в пространстве (для любого $r$ ), если $u \in L^{2}\left(\mathbb{R}^{3}\right)$ (см. (34)), а $\|\nabla \beta\|_{L^{2}\left(\mathbb{R}^{3}\right)}$ оценивается через $\|u\|_{L^{4}\left(\mathbb{R}^{3}\right)}^{2}($ см. (47)). С другой стороны, $\pi$ при малых $r$ имеет порядок $r^{2}|\nabla u|^{2}$. Для 3-мерных уравнений Навье-Стокса несжимаемой жидкости хорошо известны критерии регулярности [1], [7]. Если бы давление удовлетворяло тем же оценкам, что и $\beta$, отсюда легко следовала бы регулярность решений 3-мерных уравнений Навье-Стокса. Поскольку $\pi(x, r) \rightarrow 0$ при $r \rightarrow 0$, предположение, что $p$ удовлетворяет тем же оценкам, что и $\beta$, вполне осмысленно. С другой стороны, для оценок $\pi$ нам требуется некоторая гладкость поля скоростей. Для слабых решений 3-мерных уравнений Навье-Стокса повышение регулярности скорости по пространственным переменным было получено в [4] (см. также [9]). Эти оценки означают, что $\pi(x, r)$ остается малым почти все время. $\mathrm{K}$ примеру, $\|\pi\|_{L^{3}\left(\mathbb{R}^{3}\right)} \leqslant C(t) r^{2} t$-почти всюду (см. $(52),(59))$. Проблема в том, что, вообще говоря, $C(t)$ недостаточно хорошо интегрируется по времени, чтобы можно было сделать вывод о регулярности $\left(C(t)^{1 / 3}\right.$ интегрируемо по времени, а для регулярности достаточно было бы интегрируемости $C(t))$.

Статья построена следующим образом. В разделе 2 представлены основные вычисления, которые приводят к формулам для давления. В разделе 3 мы даем вытекающие из них оценки для $\eta$ и $\pi$. В разделе 4 излагается короткое доказательство оценок старших производных решений трехмерных уравнений Навье-Стокса во всем пространстве. Они вытекают из классической работы [4] и известны уже несколько десятилетий, хотя, поскольку в [4] рассматривались 
пространственно периодические решения, доказательство одного из результатов ${ }^{1}$ для всего пространства было получено только в 2001 г. [2]. Доказательство этого результата и ряд ссылок также можно найти в [8] (2012 г.). В разделе 5 приводятся два применения: первым из них является простое доказательство того факта, что если $u \in C^{\alpha}$, то $p \in C^{2 \alpha}$ (при $2 \alpha<1$; если же $2 \alpha>1$, то $p$ оказывается липшицевой функцией). Этот результат был недавно использован в [5], где его доказательство основывалось на разложении Литтлвуда-Пэли. Ранее Л. Сильвестром было получено еще одно доказательство (более похожее на наше), которое, однако, не было опубликовано. Решение 3-мерных уравнений Навье-Стокса называется регулярным, если $u \in L^{\infty}\left([0, T], L^{3}\left(\mathbb{R}^{3}\right)\right)[3],[6]$. В качестве второго применения мы приводим критерии регулярности для 3 -мерных уравнений Навье-Стокса, формулируемые в терминах $\pi$. Фактически они говорят, что если можно найти малое $r(t)$, для которого $\pi$ также мало в некотором смысле, а некий интеграл от $r(t)^{-1}$ конечен, то имеет место регулярность.

Некоторые элементарные вычисления, необходимые для вывода формул, приводятся в приложении (раздел 6).

\section{2. Сферические средние}

Положим

$$
\bar{f}(x, r)=\frac{1}{4 \pi r^{2}} \int_{|x-y|=r} f(y) d S(y)=f_{|\xi|=1} f(x+r \xi) d S(\xi),
$$

где $f$ - нормализованный интеграл. Рассматриваются решения уравнения

$$
-\Delta p=\nabla \cdot(u \cdot \nabla u)
$$

в $\Omega \subset \mathbb{R}^{3}$. Мы предполагаем, что $\nabla \cdot u=0$ и что $u$ - гладкая функция. Сначала вычислим

$$
\begin{aligned}
\partial_{r} \bar{p}(x, r) & =f_{|\xi|=1} \xi \cdot \nabla_{x} p(x+r \xi) d S(\xi)=\frac{1}{4 \pi r} \int_{|\xi|=1} \xi \cdot \nabla_{\xi} p(x+r \xi) d S(\xi) \\
& =\frac{1}{4 \pi r} \int_{|\xi|<1} \Delta_{\xi} p(x+r \xi) d \xi=\frac{r}{4 \pi} \int_{|\xi|<1} \Delta_{x} p(x+r \xi) d \xi .
\end{aligned}
$$

Будем использовать уравнение (2). Заметим, что ввиду несжимаемости, выраженной условием $\nabla \cdot u=0$,

$$
\Delta p=-\partial_{i} \partial_{j}\left(\left(u_{i}-v_{i}\right)\left(u_{j}-v_{j}\right)\right)
$$

для всякого постоянного вектора $v$ (мы придерживаемся стандартного соглашения о суммировании по повторяющимся индексам, если не оговорено про-

\footnotetext{
${ }^{1}$ исходно принадлежащего Люку Тартару; см. благодарность в [4].
} 
тивное). Таким образом,

$$
\begin{aligned}
\partial_{r} \bar{p}(x, r) & =-\frac{r}{4 \pi} \int_{|\xi|<1} \partial_{i} \partial_{j}\left(\left(u_{i}-v_{i}\right)\left(u_{j}-v_{j}\right)\right)(x+r \xi) d \xi \\
& =-\frac{1}{4 \pi} \int_{|\xi|<1} \partial_{\xi_{i}} \partial_{j}\left(\left(u_{i}-v_{i}\right)\left(u_{j}-v_{j}\right)\right)(x+r \xi) d \xi \\
& =-\frac{1}{4 \pi} \int_{|\xi|=1} \xi_{i} \partial_{j}\left(\left(u_{i}-v_{i}\right)\left(u_{j}-v_{j}\right)\right)(x+r \xi) d S(\xi) \\
& =-\frac{1}{4 \pi r} \int_{|\xi|=1} \xi_{i} \partial_{\xi_{j}}\left(\left(u_{i}-v_{i}\right)\left(u_{j}-v_{j}\right)\right)(x+r \xi) d S(\xi) .
\end{aligned}
$$

Итак,

$$
r \partial_{r} \bar{p}(x, r)=-f_{|\xi|=1} \xi_{i} \partial_{\xi_{j}}\left(\left(u_{i}-v_{i}\right)\left(u_{j}-v_{j}\right)\right)(x+r \xi) d S(\xi) .
$$

Лемма 1. Пусть $\Omega$ - открытое множество в $\mathbb{R}^{3}$, a $x \in \Omega$. Предположим, что $r<\operatorname{dist}(x, \partial \Omega)$, и пусть $и$ - бездивергентное векторное поле из $C^{2}(\Omega)^{3}$. Пусть $v \in \mathbb{R}^{3}$. Предположим, что р решает уравнение (2) в $\Omega$. Тогда

$$
\begin{aligned}
& \partial_{r}\left\{\bar{p}(x, r)+f_{|\xi|=1}|\xi \cdot(u(x+r \xi)-v)|^{2} d S(\xi)\right\} \\
& \quad=-\frac{1}{r} f_{|\xi|=1}\left[3|\xi \cdot(u(x+r \xi)-v)|^{2}-|u(x+r \xi)-v|^{2}\right] d S(\xi) .
\end{aligned}
$$

ДокАЗАТЕЛьство. Воспользуемся тождествами

$$
\begin{aligned}
f_{|\xi|=1} \xi_{j} \partial_{\xi_{j}} f(x+r \xi) d S(\xi)=r \partial_{r} & {\left[f_{|\xi|=1} \xi_{j}^{2} f(x+r \xi) d S(\xi)\right] } \\
& +f_{|\xi|=1}\left(3 \xi_{j}^{2}-1\right) f(x+r \xi) d S(\xi)
\end{aligned}
$$

для любого $j$ (в этой формуле мы не суммируем по повторяющимся индексам) и

$$
\begin{aligned}
f_{|\xi|=1}\left(\xi_{i} \partial_{\xi_{j}}+\xi_{j} \partial_{\xi_{i}}\right) f(x+r \xi) d S(\xi)=r \partial_{r} & {\left[f_{|\xi|=1} 2 \xi_{i} \xi_{j} f(x+r \xi) d S(\xi)\right] } \\
& +f_{|\xi|=1} 6 \xi_{i} \xi_{j} f(x+r \xi) d S(\xi) .
\end{aligned}
$$

Доказательства этих тождеств элементарны и полностью приводятся в разделе 6. Ввиду (3), выражение, которое мы должны усреднить, имеет (с точностью до знака) вид

$$
\begin{array}{r}
\xi_{1} \partial_{\xi_{1}} w_{1}^{2}+\xi_{2} \partial_{\xi_{2}} w_{2}^{2}+\xi_{3} \partial_{\xi_{3}} w_{3}^{2}+\left(\xi_{1} \partial_{\xi_{2}}+\xi_{2} \partial_{\xi_{1}}\right)\left(w_{1} w_{2}\right) \\
+\left(\xi_{1} \partial_{\xi_{3}}+\xi_{3} \partial_{\xi_{1}}\right)\left(w_{1} w_{3}\right)+\left(\xi_{2} \partial_{\xi_{3}}+\xi_{3} \partial_{\xi_{2}}\right)\left(w_{2} w_{3}\right)
\end{array}
$$

где $w=u-v$, а усреднение проводится по $x+r \xi$. С помощью (5) и (6) сгруппируем и сложим члены, содержащие $r \partial_{r}$, и отдельно сгруппируем те, что не содержат $r \partial_{r}$. Тогда из (3) получаем

$$
r \partial_{r} \bar{p}(x, r)=-r \partial_{r} f_{|\xi|=1}(\xi \cdot w)^{2} d S(\xi)-f_{|\xi|=1}\left[3(\xi \cdot w)^{2}-|w|^{2}\right] d S(\xi),
$$

что эквивалентно (4). 
Лемма 2. Для $x \in \Omega \subset \mathbb{R}^{3}$ пусть $0<r<\operatorname{dist}(x, \partial \Omega)$, и предположим, что $p$ - решение уравнения (2) для бездивергентного поля $u \in C^{2}(\Omega)^{3}$. Пусть $v \in \mathbb{R}^{3}$. Тогда

$$
\begin{aligned}
p(x) & +\frac{1}{3}|u(x)-v|^{2}=\bar{p}(x, r)+f_{|\xi|=1}|\xi \cdot(u(x+r \xi)-v)|^{2} d S(\xi) \\
& +\int_{0}^{r} \frac{d \rho}{\rho} f_{|\xi|=1}\left[3|\xi \cdot(u(x+\rho \xi)-v)|^{2}-|u(x+\rho \xi)-v|^{2}\right] d S(\xi) .
\end{aligned}
$$

ДокАзАтельство. Это утверждение непосредственно следует из (4) интегрированием $\int_{0}^{r} d \rho$, если заметить, что

$$
\bar{p}(x, 0)=p(x)
$$

и

$$
\lim _{r \rightarrow 0} f_{|\xi|=1}|\xi \cdot(u(x+r \xi)-v)|^{2} d S(\xi)=\frac{1}{3} \lim _{r \rightarrow 0} f_{|\xi|=1}|u(x+r \xi)-v|^{2} d S(\xi) .
$$

Формулу (8) можно конкретизировать, подбирая $v$. Однако сначала введем величину

$$
\sigma_{i j}(\widehat{y-x})=3 \frac{\left(y_{i}-x_{i}\right)\left(y_{j}-x_{j}\right)}{|y-x|^{2}}-\delta_{i j}
$$

где

$$
\widehat{y-x}=\frac{y-x}{|y-x|}
$$

Заметим, что

$$
\partial_{i} \partial_{j}\left(\frac{1}{|x-y|}\right)=\frac{\sigma_{i j}(\widehat{y-x})}{|y-x|^{3}} .
$$

Теперь, взяв $v=0$ в (8), получаем

$$
\begin{aligned}
p(x)+\frac{1}{3}|u(x)|^{2}= & \bar{p}(x, r)+f_{|y-x|=r}|\xi \cdot u(y)|^{2} d S(y) \\
& +\frac{1}{4 \pi} \mathrm{PV} \int_{B(x, r)} \frac{\sigma_{i j}(\widehat{x-y})}{|x-y|^{3}}\left(u_{i} u_{j}\right)(y) d y .
\end{aligned}
$$

ЗАмЕЧАНИЕ 3. При $\Omega=\mathbb{R}^{3}$, взяв интеграл $R^{-1} \int_{R}^{2 R} d r$ и положив $R \rightarrow \infty$ в (12), получаем (в предположении, что $R^{-1} \int_{R}^{2 R} \bar{p} d r$ стремится к нулю)

$$
p(x)+\frac{1}{3}|u(x)|^{2}=\frac{1}{4 \pi} \mathrm{PV} \int_{\mathbb{R}^{3}} \frac{\sigma_{i j}(\widehat{x-y})}{|x-y|^{3}}\left(u_{i} u_{j}\right)(y) d y ;
$$

это также можно вывести из равенства

$$
p(x)=\frac{1}{4 \pi} \int_{\mathbb{R}^{3}} \frac{1}{|x-y|} \partial_{i} \partial_{j}\left(u_{i} u_{j}\right)(y) d y
$$

путем интегрирования по частям. Таким образом, (12) является локальным вариантом формулы (13), верным для всякого $r>0$. 
Взяв $v=u(x)$ в $(8)$, получим

$$
\begin{aligned}
p(x) & -\bar{p}(x, r)-f_{|y-x|=r}|\xi \cdot(u(y)-u(x))|^{2} d S(y) \\
& =\frac{1}{4 \pi} \int_{B(x, r)} \frac{\sigma_{i j}(\widehat{x-y})}{|x-y|^{3}}\left(u_{i}(y)-u_{i}(x)\right)\left(u_{j}(y)-u_{j}(x)\right) d y .
\end{aligned}
$$

Чтобы прояснить связь между (12) и (14), заметим, что

$$
f_{|y-x|=r} \xi_{i}(\xi \cdot u(y)) d S(y)+\frac{1}{4 \pi} \mathrm{PV} \int_{B(x, r)} \frac{\sigma_{i j}(\widehat{x-y})}{|x-y|^{3}} u_{j}(y) d y=\frac{1}{3} u_{i}(x) .
$$

Это следует из очевидного равенства

$$
\frac{1}{4 \pi} \int_{B(x, r)} \frac{y_{i}-x_{i}}{|y-x|^{3}}(\nabla \cdot u)(y) d y=0
$$

после интегрирования по частям.

ЗАмЕчАНиЕ 4. Положив $r \rightarrow \infty$, в случае всего пространства для убывающей к нулю $u$ из (15) можно вывести, что

$$
\frac{1}{4 \pi} \mathrm{PV} \int_{\mathbb{R}^{3}} \frac{\sigma_{i j}(\widehat{x-y})}{|x-y|^{3}} u_{j}(y) d y=\frac{1}{3} u_{i}(x)
$$

это также следует из равенства $\mathbb{P} u=u$, где $\mathbb{P}$ - проектор на бездивергентные функции, действующий по формуле

$$
\mathbb{P} v=\frac{2}{3} v+\frac{1}{4 \pi} \mathrm{PV} \int_{\mathbb{R}^{3}} \frac{\sigma_{i j}(\widehat{x-y})}{|x-y|^{3}} v_{j}(y) d y .
$$

Теперь в интеграле в смысле главного значения в формуле (12) запишем

$$
\begin{aligned}
u_{i}(y) u_{j}(y)=( & \left.u_{i}(y)-u_{i}(x)\right)\left(u_{j}(y)-u_{j}(x)\right) \\
& +u_{i}(x) u_{j}(y)+u_{j}(x) u_{i}(y)-u_{i}(x) u_{j}(x)
\end{aligned}
$$

и воспользуемся тем, что средние от $\sigma_{i j}(\widehat{y-x}) /|y-x|^{3}$ по сферам с центром в $x$ обращаются в нуль. Тогда с помощью (15) мы получим

$$
\begin{aligned}
p(x) & +\frac{1}{3}|u(x)|^{2}=\bar{p}(x, r)+f_{|y-x|=r}|\xi \cdot u(y)|^{2} d S(y) \\
& +\frac{1}{4 \pi} \mathrm{PV} \int_{B(x, r)} \frac{\sigma_{i j}(\widehat{x-y})}{|x-y|^{3}}\left(u_{i}(y)-u_{i}(x)\right)\left(u_{j}(y)-u_{j}(x)\right) d y \\
& -2 f_{|y-x|=r}(\xi \cdot u(x))(\xi \cdot u(y)) d S(y)+\frac{2}{3}|u(x)|^{2} .
\end{aligned}
$$


Переставляя члены и замечая, что

$$
f_{|y-x|=r}(\xi \cdot u(x))^{2} d S(y)=\frac{1}{3}|u(x)|^{2},
$$

в итоге получим равенство

$$
\begin{aligned}
p(x)=\bar{p}(x, r) & +f_{|y-x|=r}|\xi \cdot(u(y)-u(x))|^{2} d S(y) \\
& +\frac{1}{4 \pi} \int_{B(x, r)} \frac{\sigma_{i j}(\widehat{x-y})}{|x-y|^{3}}\left(u_{i}(y)-u_{i}(x)\right)\left(u_{j}(y)-u_{j}(x)\right) d y .
\end{aligned}
$$

Итак, мы можем сделать следующее замечание.

ЗАмЕчАниЕ 5. Формула (14) выводится непосредственно из (12) с помощью формулы (15), которая вытекает из бездивергентности.

ЗАмечАниЕ 6. Ситуация в $\mathbb{R}^{2}$ совершенно аналогична. Вместо (5) и (6) выполнены соотношения

$$
\begin{aligned}
f_{\mathbb{S}^{1}} \xi_{j} \partial_{\xi_{j}} f(x+r \xi) d S(\xi)=r \partial_{r} & f_{\mathbb{S}^{1}} \xi_{j}^{2} f(x+r \xi) d S(\xi) \\
& +f_{\mathbb{S}^{1}}\left(2 \xi_{j}^{2}-1\right) f(x+r \xi) d S(\xi)
\end{aligned}
$$

для $j=1,2$, а также

$$
\begin{aligned}
& f_{\mathbb{S}^{1}}\left(\xi_{1} \partial_{\xi_{2}}+\xi_{2} \partial_{\xi_{1}}\right) f(x+r \xi) d S(\xi) \\
& \quad=r \partial_{r} f_{\mathbb{S}^{1}} 2 \xi_{1} \xi_{2} f(x+r \xi) d S(\xi)+f_{\mathbb{S}^{1}} 2 \xi_{1} \xi_{2} f(x+r \xi) d S(\xi),
\end{aligned}
$$

так что вместо (7) получается равенство

$$
r \partial_{r} \bar{p}(x, r)=-r \partial_{r} f_{|\xi|=1}(\xi \cdot w)^{2} d S(\xi)-f_{|\xi|=1}\left[2(\xi \cdot w)^{2}-|w|^{2}\right] d S(\xi),
$$

в котором $w=u(x+r \xi)-v$, а $v-$ постоянный вектор. Это равенство снова приводит к локальному представлению

$$
\begin{aligned}
p(x) & +\frac{1}{2}|u(x)-v|^{2}=\bar{p}(x, r)+f_{|\xi|=1}|\xi \cdot(u(x+r \xi)-v)|^{2} d S(\xi) \\
& +\int_{0}^{r} \frac{d \rho}{\rho} f_{|\xi|=1}\left[2|\xi \cdot(u(x+\rho \xi)-v)|^{2}-|u(x+\rho \xi)-v|^{2}\right] d S(\xi) .
\end{aligned}
$$

Мы завершим этот раздел указанием на аналогичные формулы для среднего значения градиента давления. К примеру, отправляясь от замечания, что $\partial_{1} p-$ решение уравнения

$$
-\Delta \partial_{1} p=\partial_{i} \partial_{j}\left(\partial_{1}\left(u_{i} u_{j}\right)\right)
$$

полученного дифференцированием (2), мы приходим к равенству

$$
\partial_{r} \overline{\partial_{1} p}=-\partial_{r} f_{|\xi|=1} \xi_{i} \xi_{j}\left(\partial_{x_{1}}\left(u_{i} u_{j}\right)(x+r \xi)\right) d S(\xi)
$$




$$
\begin{aligned}
& -\frac{1}{r} f_{|\xi|=1}\left(3 \xi_{i} \xi_{j}-\delta_{i j}\right)\left(\partial_{x_{1}}\left(u_{i} u_{j}\right)(x+r \xi)\right) d S(\xi) \\
=- & \partial_{r}\left(\frac{1}{r} f_{|\xi|=1} \xi_{i} \xi_{j}\left(\partial_{\xi_{1}}\left(u_{i} u_{j}\right)(x+r \xi)\right) d S(\xi)\right) \\
& -\frac{1}{r^{2}} f_{|\xi|=1}\left(3 \xi_{i} \xi_{j}-\delta_{i j}\right)\left(\partial_{\xi_{1}}\left(u_{i} u_{j}\right)(x+r \xi)\right) d S(\xi) .
\end{aligned}
$$

В (23) мы интегрируем по частям, используя соотношения

$$
\begin{aligned}
& f_{|\xi|=1} \xi_{1} \xi_{2} \partial_{\xi_{1}} f(x+r \xi) d S(\xi)=r \partial_{r} f \xi_{1}^{2} \xi_{2} f(x+r \xi) d S(\xi) \\
& +f_{|\xi|=1}\left(4 \xi_{1}^{2}-1\right) \xi_{2} f(x+r \xi) d S(\xi), \\
& f_{|\xi|=1} \xi_{1} \xi_{3} \partial_{\xi_{1}} f(x+r \xi) d S(\xi)=r \partial_{r} f \xi_{1}^{2} \xi_{3} f(x+r \xi) d S(\xi) \\
& +f_{|\xi|=1}\left(4 \xi_{1}^{2}-1\right) \xi_{3} f(x+r \xi) d S(\xi), \\
& f_{|\xi|=1} \xi_{1}^{2} \partial_{\xi_{1}} f(x+r \xi) d S(\xi)=r \partial_{r} f \xi_{1}^{3} f(x+r \xi) d S(\xi) \\
& +f_{|\xi|=1}\left(4 \xi_{1}^{2}-2\right) \xi_{1} f(x+r \xi) d S(\xi), \\
& f_{|\xi|=1} \xi_{2}^{2} \partial_{\xi_{1}} f(x+r \xi) d S(\xi)=r \partial_{r} f \xi_{1} \xi_{2}^{2} f(x+r \xi) d S(\xi) \\
& +f_{|\xi|=1} 4 \xi_{1} \xi_{2}^{2} f(x+r \xi) d S(\xi) \\
& f_{|\xi|=1} \xi_{3}^{2} \partial_{\xi_{1}} f(x+r \xi) d S(\xi)=r \partial_{r} f \xi_{1} \xi_{3}^{2} f(x+r \xi) d S(\xi) \\
& +f_{|\xi|=1} 4 \xi_{1} \xi_{3}^{2} f(x+r \xi) d S(\xi) \\
& f_{|\xi|=1} \xi_{2} \xi_{3} \partial_{\xi_{1}} f(x+r \xi) d S(\xi)=r \partial_{r} f \xi_{1} \xi_{2} \xi_{3} f(x+r \xi) d S(\xi) \\
& +f_{|\xi|=1} 4 \xi_{1} \xi_{2} \xi_{3} f(x+r \xi) d S(\xi)
\end{aligned}
$$

доказательство которых схоже с доказательством тождеств (5), (6). Произведя вычисления с использованием этих соотношений, мы получаем

$$
\begin{aligned}
\partial_{r} \overline{\partial_{1} p}=- & {\left[\partial_{r}^{2}+\frac{7}{r} \partial_{r}+\frac{8}{r^{2}}\right] f_{|\xi|=1} \xi_{1}(\xi \cdot u(x+r \xi))^{2} d S(\xi) } \\
& +\frac{2}{r}\left[\partial_{r}+\frac{2}{r}\right] f_{|\xi|=1} u_{1}(x+r \xi)(\xi \cdot u(x+r \xi)) d S(\xi) \\
& +\frac{1}{r}\left[\partial_{r}+\frac{2}{r}\right] f_{|\xi|=1} \xi_{1}|u(x+r \xi)|^{2} d S(\xi)
\end{aligned}
$$


Это равенство выполняется, поскольку

$$
\overline{\xi_{i} \xi_{j} \partial_{\xi_{1}} u_{i} u_{j}}=\left[r \partial_{r}+4\right] \overline{\xi_{1}(\xi \cdot u)^{2}}-2 \overline{u_{1}(\xi \cdot u)}
$$

и

$$
\overline{\partial_{\xi_{1}}|u(x+r \xi)|^{2}}=\left[r \partial_{r}+2\right] \overline{\xi_{1}|u|^{2}} .
$$

\section{3. Представление и оценки}

В этом разделе $\Omega=\mathbb{R}^{3}$. Рассмотрим

$$
b(x, r)=\bar{p}(x, r)+f_{|\xi|=1}|\xi \cdot u(x+r \xi)|^{2} d S(\xi) .
$$

Уравнение (4) с $v=0$ имеет вид

$$
\partial_{r} b(x, r)=r^{-1}\left[\overline{|u|^{2}-3|\xi \cdot u(y)|^{2}}\right](x, r),
$$

и, интегрируя от $r$ до бесконечности и вспомнив (11), мы получаем

$$
b(x, r)=-\frac{1}{4 \pi} \int_{|x-y| \geqslant r} \frac{\sigma_{i j}(\widehat{x-y})}{|x-y|^{3}} u_{i}(y) u_{j}(y) d y .
$$

УтвеРЖДЕНИЕ 7. Пусть $x \in \mathbb{R}^{3}, r>0, u$ предположим, что $p$ - решение уравнения (2) в $\Omega=\mathbb{R}^{3}$ с бездивергентной $u \in\left(C^{2}\left(\mathbb{R}^{3}\right) \cap L^{2}\left(\mathbb{R}^{3}\right)\right)^{3}$. Пусть ь задано в (28). Тогда

$$
\sup _{x \in \mathbb{R}^{3}}|b(x, r)| \leqslant \frac{1}{2 \pi r^{3}}\|u\|_{L^{2}}^{2} \text {. }
$$

Если $u \in H^{1}\left(\mathbb{R}^{3}\right)$, mo

$$
\sup _{x \in \mathbb{R}^{3}}|b(x, r)| \leqslant \frac{C}{2 \pi r}\|\nabla u\|_{L^{2}}^{2},
$$

где $C$ - константа из неравенства Харди в $\mathbb{R}^{3}$.

ЗАмечАниЕ 8 . Очевидно, что от функции $u$ нам не требуется $C^{2}$-гладкость, но требуется достаточная регулярность, чтобы $b$ можно было определить формулой (28). Разумеется, для представления (30) нужно только, чтобы $u \in L^{2}$.

ЗАмЕчАниЕ 9. Соответствующим локальным результатом в открытом множестве $\Omega$ является оценка $b(\cdot, r)$ в $L^{\infty}(d x)$ через локальные $L^{1}(d x)$-оценки для $b$ и $L^{2}$ - (или $H^{1}$-) оценки для $u$. Ее можно вывести непосредственно, умножив (29) на подходящую финитную функцию от $r$ и проинтегрировав по $r$.

ДокАЗАТЕЛЬСтво УТвЕРЖДЕНИЯ 7. Это сразу следует из неравенства

$$
\left|\sigma_{i j}(\xi) u_{i} u_{j}\right| \leqslant 2|u|^{2}
$$

выполненного для любых $u \in \mathbb{R}^{3}$ и $\xi \in \mathbb{S}^{2}$, и неравенства Харди

$$
\int_{\mathbb{R}^{3}} \frac{|u(y)|^{2}}{|x-y|^{2}} d y \leqslant C \int_{\mathbb{R}^{3}}|\nabla u(y)|^{2} d y .
$$

Теперь положим

$$
\beta(x, r)=\frac{1}{r} \int_{r}^{2 r} \bar{p}(x, \rho) d \rho .
$$


УтвеРЖДЕНИЕ 10. Пусть $x \in \mathbb{R}^{3}, r>0$, и предположим, что $p$ - решение уравнения (2) в $\Omega=\mathbb{R}^{3}$ с бездивергентной $u \in\left(C^{2}\left(\mathbb{R}^{3}\right) \cap L^{2}\left(\mathbb{R}^{3}\right)\right)^{3}$. Пусть $\beta$ определено в (33). Тогда

$$
\sup _{x \in \mathbb{R}^{3}}|\beta(x, r)| \leqslant \frac{3}{4 \pi r^{3}}\|u\|_{L^{2}}^{2}
$$

Eсли $u \in H^{1}\left(\mathbb{R}^{3}\right)$, mo

$$
\sup _{x \in \mathbb{R}^{3}}|\beta(x, r)| \leqslant \frac{3 C}{4 \pi r}\|\nabla u\|_{L^{2}}^{2},
$$

где С - константа из неравенства Харди в $\mathbb{R}^{3}$.

ДокАЗАТЕльство. Заметим, что

$$
\beta(x, r)=\frac{1}{r} \int_{r}^{2 r}\left(b(x, \rho)-\overline{(\xi \cdot u)^{2}}(x, \rho)\right) d \rho .
$$

Доказываемые неравенства немедленно вытекают из равенства

$$
\frac{1}{r} \int_{r}^{2 r} \overline{(\xi \cdot u)^{2}}(x, \rho) d \rho=\frac{1}{4 \pi r} \int_{r \leqslant|x-y| \leqslant 2 r}\left(\frac{x-y}{|x-y|} \cdot u(y)\right)^{2} \frac{d y}{|x-y|^{2}},
$$

утверждения 7 и неравенства Харди.

ЗАмечАниЕ 11 . Мы вводим средние $\beta(x, r)$ от величины $\bar{p}(x, r)$ для того, чтобы от поточечной информации о $b(x, r)(31),(32)$ перейти к поточечной информации о $\beta(x, r)(34),(35)$, не накладывая других условий, кроме $L^{2}$ (или $H^{1}{ }^{-}$) оценок для $u$.

Введем весовую функцию

$$
w(\lambda)= \begin{cases}1, & 0 \leqslant \lambda \leqslant 1 \\ 2-\lambda, & 1 \leqslant \lambda \leqslant 2 \\ 0, & \lambda \geqslant 2\end{cases}
$$

Теперь используем представление (14) и среднее значение по $r$. Мы приходим к следующему утверждению.

Tеорема 12. Пусть $x \in \mathbb{R}^{3}, r>0$, и предоложим, что $p$ - решение уравнения (2) в $\Omega=\mathbb{R}^{3}$ с бездивергентной $u \in\left(C^{2}\left(\mathbb{R}^{3}\right) \cap L^{2}\left(\mathbb{R}^{3}\right)\right)^{3}$. Тогда

$$
p(x)=\beta(x, r)+\pi(x, r),
$$

где $\beta(x, r)$ задано формулой

$$
\beta(x, r)=\frac{1}{r} \int_{r}^{2 r} \bar{p}(x, \rho) d \rho
$$

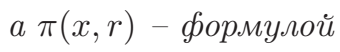

$$
\begin{aligned}
& \pi(x, r)=\frac{1}{4 \pi r} \int_{r \leqslant|y-x| \leqslant 2 r} \frac{1}{|y-x|^{2}}\left(\frac{y-x}{|y-x|} \cdot(u(y)-u(x))\right)^{2} d y \\
& +\frac{1}{4 \pi} \int_{|x-y| \leqslant 2 r} w\left(\frac{|y-x|}{r}\right) \frac{\sigma_{i j}(\widehat{x-y})}{|x-y|^{3}}\left(u_{i}(y)-u_{i}(x)\right)\left(u_{j}(y)-u_{j}(x)\right) d y .
\end{aligned}
$$


ЗАмЕчАниЕ 13 . Переходя в (37) к пределу при $r \rightarrow \infty$, получаем равенство

$$
p(x)=\frac{|u(x)|^{2}}{3}+\frac{1}{4 \pi} \int_{\mathbb{R}^{3}} \frac{\sigma_{i j}(\widehat{z})}{|z|^{3}}\left(u_{i}(x+z)-u_{i}(x)\right)\left(u_{j}(x+z)-u_{j}(x)\right) d z .
$$

Его можно также вывести из (13), используя (16).

ДокАЗАТЕЛЬство теоремы 12 . Возьмем интеграл $\frac{1}{r} \int_{r}^{2 r} d \rho$ от представления (14), записанного в виде

$$
\begin{aligned}
p(x)= & \bar{p}(x, \rho)+f_{|y-x|=\rho}|\xi \cdot(u(y)-u(x))|^{2} d S(y) \\
& +\int_{0}^{\rho} \frac{d l}{l} f_{|y-x|=l}\left[3(\xi \cdot(u(y)-u(x)))^{2}-|u(y)-u(x)|^{2}\right] d S(y),
\end{aligned}
$$

и используем равенство

$$
\frac{1}{r} \int_{r}^{2 r}\left(\int_{0}^{\rho} f(l) d l\right) d \rho=\int_{0}^{2 r} w\left(\frac{l}{r}\right) f(l) d l .
$$

Помимо (34) и (35), у нас еще есть оценки, вытекающие из неравенства Морри

$$
\int_{\mathbb{R}^{3}}|u(y)|^{6} d y \leqslant C\left[\int_{\mathbb{R}^{3}}|\nabla u(y)|^{2} d y\right]^{3},
$$

представления

$$
p=R_{i} R_{j}\left(u_{i} u_{j}\right)
$$

для давления, где $R_{i}=\partial_{i}(-\Delta)^{-1 / 2}-$ преобразования Рисса, и ограниченности преобразования Рисса в пространствах $L^{p}$.

УтвеРЖДЕНИЕ 14. Пусть $p$ - решение уравнения (2), заданное в (42). Для всякого $q, 1<q<\infty$, существуют такие константы $C_{q}>0$, не зависящие от $r>0$, что для всякого $r>0$

$$
\|\bar{p}(\cdot, r)\|_{L^{q}\left(\mathbb{R}^{3}\right)} \leqslant C_{q}\|u\|_{L^{2 q}\left(\mathbb{R}^{3}\right)}^{2}
$$

u

$$
\|\beta(\cdot, r)\|_{L^{q}\left(\mathbb{R}^{3}\right)} \leqslant C_{q}\|u\|_{L^{2 q}\left(\mathbb{R}^{3}\right)}^{2} .
$$

Для любого $a \in[0,2)$ имеется константа $C_{a}>0$, с которой выполнено

$$
\|\beta(\cdot, r)\|_{L^{3}\left(\mathbb{R}^{3}\right)} \leqslant C_{a} r^{-a}\|u\|_{L^{2}\left(\mathbb{R}^{3}\right)}^{a}\|\nabla u\|_{L^{2}\left(\mathbb{R}^{3}\right)}^{2-a} .
$$

Существует такая константа $C>0$, ито

$$
\|\nabla \bar{p}(\cdot, r)\|_{L^{2}\left(\mathbb{R}^{3}\right)} \leqslant C r^{-1}\|u\|_{L^{4}\left(\mathbb{R}^{3}\right)}^{2}
$$

u

$$
\|\nabla \beta(\cdot, r)\|_{L^{2}\left(\mathbb{R}^{3}\right)} \leqslant C r^{-1}\|u\|_{L^{4}\left(\mathbb{R}^{3}\right)}^{2} .
$$


ДокАЗАТЕльство. Оценки (44) для $\beta$ получаются из оценок (43) для $\bar{p}$ с помощью усреднения по $r$. Оценки (43) следуют из (42) и ограниченности преобразования Рисса в пространствах $L^{p}$. Оценки (45) следуют из $(35)$, интерполяционного неравенства

$$
\|\beta\|_{L^{3}\left(\mathbb{R}^{3}\right)} \leqslant\|\beta\|_{L^{\infty}\left(\mathbb{R}^{3}\right)}^{a / 3}\|\beta\|_{L^{3-a}\left(\mathbb{R}^{3}\right)}^{1-a / 3},
$$

оценки (44) для $q=3-a$ :

$$
\|\beta(\cdot, r)\|_{L^{3-a}\left(\mathbb{R}^{3}\right)} \leqslant C_{a}\|u\|_{L^{6-2 a}\left(\mathbb{R}^{3}\right)}^{2},
$$

и из комбинации интерполяции с неравенством Морри:

$$
\|u\|_{L^{6-2 a}\left(\mathbb{R}^{3}\right)} \leqslant C\|u\|_{L^{2}\left(\mathbb{R}^{3}\right)}^{a /(6-2 a)}\|\nabla u\|_{L^{2}\left(\mathbb{R}^{3}\right)}^{(6-3 a) /(6-2 a)} .
$$

Оценка (47) следует из (46) усреднением по $r$. Оценка (46) следует из неравенства

$$
\|\nabla \bar{p}(\cdot, r)\|_{L^{2}\left(\mathbb{R}^{3}\right)} \leqslant C r^{-1}\|\bar{p}(\cdot, r)\|_{L^{2}\left(\mathbb{R}^{3}\right)}
$$

и оценки (43) с $q=2$, а (48) следует из тождества Планшереля и следующего замечания:

$$
\widehat{\bar{p}}(\xi, r)=\frac{\sin (r|\xi|)}{r|\xi|} \widehat{p}(\xi) .
$$

В самом деле,

$$
\begin{aligned}
\int_{\mathbb{R}^{3}} e^{-i x \cdot \xi} \bar{p}(x, r) d x & =f_{|\omega|=1} d S(\omega) \int_{\mathbb{R}^{3}} e^{-i x \cdot \xi} p(x+r \omega) d x \\
& =\widehat{p}(\xi) f_{|\omega|=1} e^{i r \xi \cdot \omega} d S(\omega)
\end{aligned}
$$

а последний интеграл удобно вычислять, выбрав систему координат, в которой $\xi$ направлено на Северный полюс:

$$
\frac{1}{4 \pi} \int_{0}^{2 \pi} d \phi \int_{0}^{\pi} e^{i r|\xi| \cos \theta} \sin \theta d \theta=\frac{\sin (r|\xi|)}{r|\xi|} .
$$

Относительно $\pi$ мы имеем следующий результат.

УтВеРЖДЕНИЕ 15. Пусть $\pi(x, r)$ задано в (39). Тогда

$$
|\pi(x, r)| \leqslant C \int_{|z| \leqslant 2 r} \frac{|u(x+z)-u(x)|^{2}}{|z|^{3}} d z .
$$

Следовательно,

$$
\|\pi(\cdot, r)\|_{L^{q}\left(\mathbb{R}^{3}\right)} \leqslant C_{q} r^{2}\|\nabla u\|_{L^{2 q}\left(\mathbb{R}^{3}\right)}^{2}
$$

для всех $1<q \leqslant \infty$. В частности, при $q=3$, с учетом неравенства Морри, имеем

$$
\|\pi(\cdot, r)\|_{L^{3}\left(\mathbb{R}^{3}\right)} \leqslant C r^{2}\|\Delta u\|_{L^{2}\left(\mathbb{R}^{3}\right)}^{2} .
$$

Также выполнена оценка

$$
\|\pi(\cdot, r)\|_{L^{q}\left(\mathbb{R}^{3}\right)} \leqslant C_{q}\|u\|_{L^{2 q}\left(\mathbb{R}^{3}\right)}^{2} .
$$


ДокАзАтельство. Неравенство (50) непосредственно следует из определения. Для доказательства (51) запишем

$$
|u(x+z)-u(x)|^{2} \leqslant|z|^{2} \int_{0}^{1}|\nabla u(x+\lambda z)|^{2} d \lambda
$$

и, поменяв порядок интегрирования, получим

$$
\left|\int_{\mathbb{R}^{3}} \phi(x) d x \int_{|z| \leqslant 2 r} \frac{|u(x+z)-u(x)|^{2}}{|z|^{3}} d z\right| \leqslant C r^{2}\|\phi\|_{L^{q^{\prime}}}\|\nabla u\|_{L^{2 q}}^{2},
$$

что и доказывает (51). Оценка (53) следует из (37), соответствующих оценок для $p$ и (44).

\section{FGT-оценки [4] во всем пространстве}

Рассмотрим уравнение Навье-Стокса

$$
\partial_{t} u+u \cdot \nabla u-\nu \Delta u+\nabla p=0,
$$

в котором

$$
\nabla \cdot u=0
$$

умножим его на $\partial_{t} u-\nu \Delta u$ и проинтегрируем с учетом несжимаемости:

$$
\int_{\mathbb{R}^{3}}\left|\partial_{t} u-\nu \Delta u\right|^{2} d x=-\int_{\mathbb{R}^{3}}(u \cdot \nabla u)\left(\partial_{t} u-\nu \Delta u\right) d x .
$$

По неравенству Коши-Буняковского-Шварца

$$
\int_{\mathbb{R}^{3}}\left|\partial_{t} u-\nu \Delta u\right|^{2} d x \leqslant \int_{\mathbb{R}^{3}}|u \cdot \nabla u|^{2} d x
$$

так что

$$
\int_{\mathbb{R}^{3}}\left|\partial_{t} u-\nu \Delta u\right|^{2} d x \leqslant\|u\|_{L^{\infty}}^{2}\|\nabla u\|_{L^{2}}^{2} .
$$

С помощью преобразования Фурье легко доказать неравенство

$$
\|u\|_{L^{\infty}}^{2} \leqslant C\|\nabla u\|_{L^{2}}\|\Delta u\|_{L^{2}}
$$

Итак,

$$
\int_{\mathbb{R}^{3}}\left|\partial_{t} u-\nu \Delta u\right|^{2} d x \leqslant C\|\Delta u\|_{L^{2}}\|\nabla u\|_{L^{2}}^{3} .
$$

С другой стороны,

$$
\int_{\mathbb{R}^{3}}\left|\partial_{t} u-\nu \Delta u\right|^{2} d x=\left\|\partial_{t} u\right\|_{L^{2}}^{2}+\nu^{2}\|\Delta u\|_{L^{2}}^{2}+\nu \frac{d}{d t}\|\nabla u\|_{L^{2}}^{2},
$$

так что

$$
\begin{aligned}
& \frac{d}{d t}\|\nabla u\|_{L^{2}}^{2}+\nu\|\Delta u\|_{L^{2}}^{2}+\frac{1}{\nu}\left\|\partial_{t} u\right\|_{L^{2}}^{2} \\
& \quad \leqslant \frac{C}{\nu}\|\Delta u\|_{L^{2}}\|\nabla u\|_{L^{2}}^{2} \leqslant \frac{\nu}{2}\|\Delta u\|_{L^{2}}^{2}+\frac{C}{\nu^{3}}\|\nabla u\|_{L^{2}}^{6} .
\end{aligned}
$$


Теперь обозначим $y(t)=\|\nabla u(\cdot, t)\|_{L^{2}}^{2}$, выберем постоянную $A>0$ и, поделив на $(A+y)^{2}$, получим

$$
-\frac{d}{d t}\left(\frac{1}{A+y}\right)+\frac{\nu\|\Delta u\|_{L^{2}}^{2}}{(A+y)^{2}}+\frac{\left\|\partial_{t} u\right\|_{L^{2}}^{2}}{\nu(A+y)^{2}} \leqslant \frac{C}{\nu^{3}} y .
$$

Интегрирование по времени дает

$$
\int_{0}^{T} \frac{\nu\|\Delta u\|_{L^{2}}^{2}}{(A+y)^{2}} d t+\int_{0}^{T} \frac{\left\|\partial_{t} u\right\|_{L^{2}}^{2}}{\nu(A+y)^{2}} d t \leqslant \frac{C}{\nu^{4}}\left\|u_{0}\right\|_{L^{2}}^{2}+\frac{1}{A} .
$$

Таким образом,

$$
\int_{0}^{T} \frac{\|\Delta u\|_{L^{2}}^{2}}{(A+y)^{2}} d t \leqslant \frac{C}{\nu^{5}}\left\|u_{0}\right\|_{L^{2}}^{2}+\frac{1}{\nu A}=C \nu^{-4}\left[D+\nu^{3} A^{-1}\right]
$$

и

$$
\int_{0}^{T} \frac{\left\|\partial_{t} u\right\|_{L^{2}}^{2}}{(A+y)^{2}} d t \leqslant \frac{C}{\nu^{3}}\left\|u_{0}\right\|_{L^{2}}^{2}+\frac{\nu}{A}=C \nu^{-2}\left[D+\nu^{-3} A^{-1}\right],
$$

где мы положили

$$
D=\frac{\left\|u_{0}\right\|_{L^{2}}^{2}}{\nu}
$$

Далее,

$$
\int_{0}^{T}\|\Delta u\|_{L^{2}}^{2 / 3} d t \leqslant\left[\int_{0}^{T} \frac{\|\Delta u\|_{L^{2}}^{2}}{(A+y)^{2}} d t\right]^{1 / 3}\left[\int_{0}^{T}(A+y) d t\right]^{2 / 3}
$$

И

$$
\int_{0}^{T}\left\|\partial_{t} u\right\|_{L^{2}}^{2 / 3} d t \leqslant\left[\int_{0}^{T} \frac{\left\|\partial_{t} u\right\|_{L^{2}}^{2}}{(A+y)^{2}} d t\right]^{1 / 3}\left[\int_{0}^{T}(A+y) d t\right]^{2 / 3}
$$

так что

$$
\int_{0}^{T}\|\Delta u\|_{L^{2}}^{2 / 3} d t \leqslant C \nu^{-4 / 3}\left[D+\nu^{3} A^{-1}\right]^{1 / 3}[D+A T]^{2 / 3}
$$

и

$$
\int_{0}^{T}\left\|\partial_{t} u\right\|_{L^{2}}^{2 / 3} d t \leqslant C \nu^{-2 / 3}\left[D+\nu^{3} A^{-1}\right]^{1 / 3}[D+A T]^{2 / 3} .
$$

Константа $A$ может быть любой, но естественно выбрать

$$
A^{2}=\nu^{3} T^{-1},
$$

тогда мы получаем

$$
\int_{0}^{T}\|\Delta u\|_{L^{2}}^{2 / 3} d t \leqslant C \nu^{-4 / 3}\left[\frac{\left\|u_{0}\right\|_{L^{2}}^{2}}{\nu}+T^{1 / 2} \nu^{3 / 2}\right]
$$

и

$$
\int_{0}^{T}\left\|\partial_{t} u\right\|_{L^{2}}^{2 / 3} d t \leqslant C \nu^{-2 / 3}\left[\frac{\left\|u_{0}\right\|_{L^{2}}^{2}}{\nu}+T^{1 / 2} \nu^{3 / 2}\right]
$$


Используя (56), мы немедленно видим, что

$$
\int_{0}^{T}\|u\|_{L^{\infty}} d t \leqslant C \nu^{-1}\left[\frac{\left\|u_{0}\right\|_{L^{2}}^{2}}{\nu}+T^{1 / 2} \nu^{3 / 2}\right]^{3 / 4}\left[\frac{\left\|u_{0}\right\|_{L^{2}}^{2}}{\nu}\right]^{1 / 4} .
$$

Теперь рассмотрим другие члены в (54). Сначала вычислим

$$
\int_{\mathbb{R}^{3}}|u \cdot \nabla u+\nabla p|^{2} d x=\|u \cdot \nabla u\|_{L^{2}}^{2}+\|\nabla p\|_{L^{2}}^{2}+2 \int_{\mathbb{R}^{3}}(u \cdot \nabla u) \cdot(\nabla p) d x .
$$

Далее,

$$
2 \int_{\mathbb{R}^{3}}(u \cdot \nabla u) \cdot(\nabla p) d x=-2 \int p \operatorname{Tr}(\nabla u)^{2} d x=2 \int_{\mathbb{R}^{3}} p \Delta p d x=-2\|\nabla p\|_{L^{2}}^{2} .
$$

Следовательно,

$$
0 \leqslant \int_{\mathbb{R}^{3}}|u \cdot \nabla u+\nabla p|^{2} d x=\|u \cdot \nabla u\|_{L^{2}}^{2}-\|\nabla p\|_{L^{2}}^{2} .
$$

С другой стороны, очевидно, выполнено неравенство

$$
\|u \cdot \nabla u\|_{L^{2}} \leqslant\|u\|_{L^{\infty}}\|\nabla u\|_{L^{2}}
$$

и, ввиду предыдущего результата,

$$
\int_{0}^{T}\|u \cdot \nabla u\|_{L^{2}}^{2 / 3} d t \leqslant C \nu^{-2 / 3}\left[\frac{\left\|u_{0}\right\|_{L^{2}}^{2}}{\nu}+T^{1 / 2} \nu^{3 / 2}\right]^{1 / 2}\left[\frac{\left\|u_{0}\right\|_{L^{2}}^{2}}{\nu}\right]^{1 / 2},
$$

а в силу неравенства $\|\nabla p\|_{L^{2}} \leqslant\|u \cdot \nabla u\|_{L^{2}}$ мы также имеем

$$
\int_{0}^{T}\|\nabla p\|_{L^{2}}^{2 / 3} d t \leqslant C \nu^{-2 / 3}\left[\frac{\left\|u_{0}\right\|_{L^{2}}^{2}}{\nu}+T^{1 / 2} \nu^{3 / 2}\right]^{1 / 2}\left[\frac{\left\|u_{0}\right\|_{L^{2}}^{2}}{\nu}\right]^{1 / 2} .
$$

Итак, мы пришли к следующей теореме.

Теорема 16. Пусть и - слабое решение по Лере уравнения Навъе-Стокса на интервале $[0, T]$. Тогда величины $\|u\|_{L^{\infty}\left(\mathbb{R}^{3}\right)},\|\Delta u\|_{L^{2}\left(\mathbb{R}^{3}\right)}^{2 / 3},\left\|\partial_{t} u\right\|_{L^{2}\left(\mathbb{R}^{3}\right)}^{2 / 3}, \| u$. $\nabla u\left\|_{L^{2}\left(\mathbb{R}^{3}\right)}^{2 / 3} \quad u\right\| \nabla p \|_{L^{2}\left(\mathbb{R}^{3}\right)}^{2 / 3}$ конечны почти всюду на интервале времени $[0, T]$, а их интегралы по времени равномерно ограничены, с оченками (59)-(63), зависящими только от $T,\left\|u_{0}\right\|_{L^{2}\left(\mathbb{R}^{3}\right)} u \nu$.

Для слабых решений по Лере доказательство проходит по той же схеме, что и приведенное выше доказательство для гладких решений, за тем исключением, что мы сглаживаем скорость адвекции, доказываем оценки, не зависящие от сглаживания, и, по сути, выводим требуемые результаты из леммы Фату. Для полноты упомянем и о других оценках. Проинтерполировав неравенства

$$
\int_{0}^{T}\|\nabla u\|_{L^{2}}^{2} d t<\infty
$$


И

$$
\int_{0}^{T}\|\nabla u\|_{L^{6}}^{2 / 3} d t<\infty
$$

(следующие из неравенства Морри и (59)), получим соотношение

$$
\|\nabla u\|_{L^{3}} \leqslant C\|\nabla u\|_{L^{6}}^{1 / 2}\|\nabla u\|_{L^{2}}^{1 / 2}
$$

которое можно проинтегрировать в силу неравенства Гёльдера:

$$
\int_{0}^{T}\|\nabla u\|_{L^{3}} d t<\infty .
$$

Наконец, отметим, что, интерполируя между $L^{\infty}\left(d t ; L^{2}(d x)\right)$ и $L^{2}\left(d t ; L^{6}(d x)\right)$, легко видеть, что $u \in L^{p}\left(d t, L^{q}(d x)\right)$ при $q=6 p /(3 p-4)$, если $p \geqslant 2$. Если же $p \in[1,2]$, то, интерполируя между $L^{2}\left(d t ; L^{6}(d x)\right)$ и $L^{1}\left(d t, L^{\infty}(d x)\right)$, получим $q=3 p /(p-1)$.

\section{5. Применения}

Теорема 17. Пусть и - решение (54) и (55) в $\mathbb{R}^{3}$, и предположим, что и принадлежст пространству $L^{\infty}\left(d t ; L^{2}\left(\mathbb{R}^{3}\right)\right) \cap L^{2 q}\left(d t ; C^{\alpha}\left(\mathbb{R}^{3}\right)\right)$ с некоторьм $q \geqslant 1$. Тогда $p \in L^{q}\left(d t ; C^{2 \alpha}\left(\mathbb{R}^{3}\right)\right)$ при $\alpha<1 / 2$. Если жее $\alpha=1 / 2$, то $p \in$ $L^{q}(d t$; LiplogLip), где LiplogLip - класс функиий с модулем непреръвности $|x-y| \log \left(|x-y|^{-1}\right)$. Если $\alpha>1 / 2$, то $p \in L^{q}(d t ; \operatorname{Lip})$, где Lip - класс функиий, непрерывных по Липшицу.

ДоказАтельство. Начнем с пары точек $x, y$ на расстоянии $|x-y|$ и положим $r=8|x-y|$. В силу представления (14),

$$
\left\{\begin{array}{l}
|p(x)-\bar{p}(x, r)| \leqslant C\|u\|_{C^{\alpha}}^{2} r^{2 \alpha}, \\
|p(y)-\bar{p}(y, r)| \leqslant C\|u\|_{C^{\alpha}}^{2} r^{2 \alpha},
\end{array}\right.
$$

так что остается доказать, что

$$
|\bar{p}(x, r)-\bar{p}(y, r)| \leqslant C r^{2 \alpha}
$$

при $2 \alpha<1$ и $C \sim\|u\|_{C^{\alpha}}^{2}$. (При $2 \alpha=1$ получаем оценку с $r \log \left(r^{-1}\right)$, а при $2 \alpha>1$ - оценку с $r$.) Для этого воспользуемся соотношением (4) с $v=u((x+$ $y) / 2$ ) и возьмем интеграл от $r$ до бесконечности. Тогда мы получим

$$
\begin{aligned}
\bar{p}(x, r)= & -f_{|\xi|=1}(\xi \cdot(u(x+r \xi)-v))^{2} d S(\xi) \\
& +\frac{1}{4 \pi} \int_{|x-z| \geqslant r} \frac{\sigma_{i j}(\widehat{x-z})}{|x-z|^{3}}\left(u_{i}(z)-v_{i}\right)\left(u_{j}(z)-v_{j}\right) d z
\end{aligned}
$$

и

$$
\begin{aligned}
\bar{p}(y, r)= & -f_{|\xi|=1}(\xi \cdot(u(y+r \xi)-v))^{2} d S(\xi) \\
& +\frac{1}{4 \pi} \int_{|y-z| \geqslant r} \frac{\sigma_{i j}(\widehat{y-z})}{|y-z|^{3}}\left(u_{i}(z)-v_{i}\right)\left(u_{j}(z)-v_{j}\right) d z .
\end{aligned}
$$


Теперь ясно, что

$$
\left|f_{|\xi|=1}(\xi \cdot(u(x+r \xi)-v))^{2} d S(\xi)\right| \leqslant C r^{2 \alpha}\|u\|_{C^{\alpha}}^{2}
$$

и

$$
\left|f_{|\xi|=1}(\xi \cdot(u(y+r \xi)-v))^{2} d S(\xi)\right| \leqslant C r^{2 \alpha}\|u\|_{C^{\alpha}}^{2},
$$

так что остается оценить

$$
\frac{1}{4 \pi} \int_{|x-z| \geqslant r} \frac{\sigma_{i j}(\widehat{x-z})}{|x-z|^{3}} w_{i} w_{j} d z-\frac{1}{4 \pi} \int_{|y-z| \geqslant r} \frac{\sigma_{i j}(\widehat{y-z})}{|y-z|^{3}} w_{i} w_{j} d z
$$

где $w=u(y)-v$. Теперь если $|x-z| \geqslant r$, но $|y-z| \leqslant r$, то $|x-z| \leqslant|y-z|+|x-y| \leqslant$ $9 r / 8$, так что

$$
\left|\frac{1}{4 \pi} \int_{|x-z| \geqslant r,|y-z| \leqslant r} \frac{\sigma_{i j}(\widehat{x-z})}{|x-z|^{3}} w_{i} w_{j} d z\right| \leqslant C\|u\|_{C^{\alpha}}^{2} r^{2 \alpha},
$$

и, аналогично, если $|y-z| \geqslant r$, но $|x-z| \leqslant r$, то

$$
\left|\frac{1}{4 \pi} \int_{|y-z| \geqslant r,|x-z| \leqslant r} \frac{\sigma_{i j}(\widehat{y-z})}{|y-z|^{3}} w_{i} w_{j} d z\right| \leqslant C\|u\|_{C^{\alpha}}^{2} r^{2 \alpha} .
$$

У нас остался интеграл

$$
\frac{1}{4 \pi} \int_{|x-z| \geqslant r,|y-z| \geqslant r}\left(K_{i j}(x-z)-K_{i j}(y-z)\right) w_{i} w_{j} d z
$$

где

$$
K_{i j}(\zeta)=\left(3 \zeta_{i} \zeta_{j}|\zeta|^{-2}-\delta_{i j}\right)|\zeta|^{-3}
$$

Это классическая задача теории сингулярных интегралов, в которой надо использовать гладкость ядра. Заметим, что

$$
\left|K_{i j}(x-z)-K_{i j}(y-z)\right| \leqslant C|x-y| \int_{0}^{1}|z-(y+\lambda(x-y))|^{-4} d \lambda
$$

и что $|z-(y+\lambda(x-y))| \geqslant 7 r / 8$. Итак,

$$
\begin{aligned}
& \left|\frac{1}{4 \pi} \int_{|x-z| \geqslant r,|y-z| \geqslant r}\left(K_{i j}(x-z)-K_{i j}(y-z)\right) w_{i} w_{j} d z\right| \\
& \quad \leqslant C|x-y| \int_{0}^{1} \int_{\left|z-x_{\lambda}\right| \geqslant 7 r / 8}\left|z-x_{\lambda}\right|^{-4}\left|u(z)-u\left(\frac{x+y}{2}\right)\right|^{2} d z d \lambda,
\end{aligned}
$$

где $x_{\lambda}=y+\lambda(x-y)$. Зафиксировав $R>0$ (можно было бы взять $R=1$, но мы хотим сохранить размерные величины), получим

$$
\begin{aligned}
& |x-y| \int_{0}^{1} \int_{\left|z-x_{\lambda}\right| \geqslant R}\left|z-x_{\lambda}\right|^{-4}\left|u(z)-u\left(\frac{x+y}{2}\right)\right|^{2} d z d \lambda \\
& \leqslant C|x-y| R^{-1}\|u\|_{L^{\infty}}^{2} .
\end{aligned}
$$


Интеграл по $7 r / 8 \leqslant\left|z-x_{\lambda}\right| \leqslant R$,

$$
|x-y| \int_{0}^{1} \int_{7 r / 8 \leqslant\left|z-x_{\lambda}\right| \leqslant R}\left|z-x_{\lambda}\right|^{-4}\left|u(z)-u\left(\frac{x+y}{2}\right)\right|^{2} d z d \lambda,
$$

можно оценить с помощью неравенства

$$
\left|u(z)-u\left(\frac{x+y}{2}\right)\right| \leqslant C\|u\|_{C^{\alpha}}^{2}\left(\left|z-x_{\lambda}\right|^{2 \alpha}+r^{2 \alpha}\right) .
$$

Проинтегрировав по $7 r / 8 \leqslant\left|z-x_{\lambda}\right| \leqslant R$, в итоге получаем оценки

$$
C\|u\|_{C^{\alpha}}^{2}|x-y|\left[\frac{1}{1-2 \alpha} r^{2 \alpha-1}+r^{2 \alpha-1}\right]
$$

для $2 \alpha<1$,

$$
C\|u\|_{C^{\alpha}}^{2}|x-y|\left[\log \left(\frac{8 R}{r}\right)+1-\frac{r}{R}\right]
$$

для $2 \alpha=1$ и

$$
C\|u\|_{C^{\alpha}}^{2}|x-y|\left[\frac{R^{2 \alpha-1}}{2 \alpha-1}+r^{2 \alpha-1}\right]
$$

для $2 \alpha>1$, что и завершает доказательство теоремы.

Теперь сформулируем несколько критериев регулярности. Через $\pi(x, t, r(t))$ будем обозначать $\pi$, определенное по формуле (39) для $u(x, t)$ и $r=r(t)$, зависящих от времени. Напомним, что если $u$ регулярна, а $r$ мало, то и $\pi$ мало.

Теорема 18. Пусть и - гладкое решение Навъе-Стокса на интервале $[0, T)$.

Первый критерий. Пусть существуют $U>0, R>0 u 0<r(t) \leqslant R, \partial л я$ которых

$$
\int_{\left\{x \in \mathbb{R}^{3}:|u(x, t)| \geqslant U\right\}}|u(x, t)||\pi(x, t, r(t))|^{2} d x \leqslant \frac{\nu^{2}}{4} \int_{\mathbb{R}^{3}}|u(x, t)||\nabla u(x, t)|^{2} d x .
$$

Пусть существует такое $\gamma>4$, что

$$
\int_{0}^{T} r(t)^{-\gamma} d t<\infty
$$

Тогда

$$
u \in L^{\infty}\left([0, T], L^{3}\left(\mathbb{R}^{3}\right)\right) .
$$

Второй критерий. Пусть существует $r(t)$, для которого $\pi=\pi(x, r(t))$ удовлетворяет соотношению

$$
\int_{0}^{T}\|\pi\|_{L^{3}\left(\mathbb{R}^{3}\right)}^{2} d t<\infty
$$

и, как и выше, существует такое $\gamma>4$, что выполнено (68). Тогда (69) снова выполнено. 
Доказательство. Сначала докажем первый критерий. Рассмотрим эволюцию $L^{3}$-нормы скорости:

$$
\frac{d}{3 d t}\|u\|_{L^{3}\left(\mathbb{R}^{3}\right)}^{3}+\nu \int_{\mathbb{R}^{3}}|\nabla u|^{2}|u| d x+\int_{\mathbb{R}^{3}}|u|(u \cdot \nabla p) d x \leqslant 0 .
$$

Представим $p$ по формуле (37) с $r=r(t)$. Разобьем интеграл, содержащий $\pi$, на два слагаемых:

$$
\begin{aligned}
\int_{\mathbb{R}^{3}}|u|(u \cdot \nabla \pi) d x= & \int_{\mathbb{R}^{3}} \phi\left(\frac{|u|}{U}\right)|u|(u \cdot \nabla \pi) d x \\
& +\int_{\mathbb{R}^{3}}\left(1-\phi\left(\frac{|u|}{U}\right)\right)|u|(u \cdot \nabla \pi) d x
\end{aligned}
$$

где $\phi(q), 0 \leqslant \phi(q) \leqslant 1,-$ гладкая скалярная функция с носителем на интервале $0 \leqslant q \leqslant 1$. Будем использовать оценку

$$
|\nabla \pi(x)| \leqslant C \int_{0}^{1} d \lambda \int_{|z| \leqslant 2 r} \frac{d z}{|z|^{2}}(|\nabla u(x+z)|+|\nabla u(x)|)|\nabla u(x+\lambda z)|,
$$

следующую из (39) дифференцированием. Тогда

$$
\left|\int_{\mathbb{R}^{3}} \phi\left(\frac{|u|}{U}\right)\right| u|(u \cdot \nabla \pi) d x| \leqslant C U^{2} r\|\nabla u\|_{L^{2}\left(\mathbb{R}^{3}\right)}^{2} .
$$

Второе слагаемое проинтегрируем по частям:

$$
\int_{\mathbb{R}^{3}}\left(1-\phi\left(\frac{|u|}{U}\right)\right)|u|(u \cdot \nabla \pi) d x=-\int_{\mathbb{R}^{3}} \pi u \cdot \nabla\left[|u|\left(1-\phi\left(\frac{|u|}{U}\right)\right)\right] d x .
$$

Когда $1-\phi$ оказывается под дифференцированием, мы оказываемся в режиме $|u| \leqslant U$ и будем использовать (53) и комбинацию интерполяции с неравенством Морри:

$$
\|u\|_{L^{4}\left(\mathbb{R}^{3}\right)}^{2} \leqslant C\|u\|_{L^{3}\left(\mathbb{R}^{3}\right)}\|\nabla u\|_{L^{2}\left(\mathbb{R}^{3}\right)},
$$

что даст нам

$$
\begin{aligned}
\left|\int_{\mathbb{R}^{3}} \pi\right| u\left|(u \cdot \nabla|u|) U^{-1} \phi^{\prime}\left(\frac{|u|}{U}\right) d x\right| & \leqslant C U\|\pi\|_{L^{2}\left(\mathbb{R}^{3}\right)}\|\nabla u\|_{L^{2}\left(\mathbb{R}^{3}\right)} \\
& \leqslant C U\|u\|_{L^{3}\left(\mathbb{R}^{3}\right)}\|\nabla u\|_{L^{2}\left(\mathbb{R}^{3}\right)}^{2} .
\end{aligned}
$$

Когда $|u|$ оказывается под дифференцированием, используем условие (67) и неравенство Коши-Буняковского-Шварца:

$$
\begin{gathered}
\left|\int_{\{|u(x, t)| \geqslant U\}} \pi(u \cdot \nabla|u|)\left(1-\phi\left(\frac{|u|}{U}\right)\right) d x\right| \\
\leqslant \frac{\nu}{2} \int_{\mathbb{R}^{3}}|u||\nabla u|^{2} d x .
\end{gathered}
$$

Обращаясь к интегралу, содержащему $\beta$, проинтегрируем по частям и используем неравенство Гёльдера, а затем (45):

$$
\left|\int_{\mathbb{R}^{3}} \beta u \cdot \nabla\right| u|d x| \leqslant\|\beta\|_{L^{3}\left(\mathbb{R}^{3}\right)}\|u\|_{L^{3}\left(\mathbb{R}^{3}\right)}^{1 / 2} \sqrt{\int_{\mathbb{R}^{3}}|u||\nabla u|^{2} d x}
$$




$$
\begin{aligned}
& \leqslant \frac{1}{2 \nu}\|\beta\|_{L^{3}\left(\mathbb{R}^{3}\right)}^{2}\|u\|_{L^{3}\left(\mathbb{R}^{3}\right)}+\frac{\nu}{2} \int_{\mathbb{R}^{3}}|u||\nabla u|^{2} d x \\
& \leqslant C \nu^{-1} r^{-2 a}\|u\|_{L^{2}\left(\mathbb{R}^{3}\right)}^{2 a}\|\nabla u\|_{L^{2}\left(\mathbb{R}^{3}\right)}^{4-2 a}\|u\|_{L^{3}\left(\mathbb{R}^{3}\right)}+\frac{\nu}{2} \int_{\mathbb{R}^{3}}|u||\nabla u|^{2} d x .
\end{aligned}
$$

Выбирая $a=\gamma /(\gamma-2)$, получим $a<2$, так что с помощью неравенства Юнга можно показать, что

$$
r^{-2 a}\|\nabla u\|_{L^{2}\left(\mathbb{R}^{3}\right)}^{4-2 a} \leqslant C\left(r^{-\gamma}+\|\nabla u\|_{L^{2}\left(\mathbb{R}^{3}\right)}^{2}\right)
$$

интегрируемо по времени. Как итог, величина $y(t)=\|u\|_{L^{3}\left(\mathbb{R}^{3}\right)}$ должна удовлетворять дифференциальному неравенству

$$
y^{2} \frac{d y}{d t} \leqslant C_{1}(t)+C_{2}(t) y+C_{3}(t) y
$$

где

$$
\begin{gathered}
C_{1}(t)=C U^{2} r\|\nabla u\|_{L^{2}\left(\mathbb{R}^{3}\right)}^{2}, \quad C_{2}(t)=C U\|\nabla u\|_{L^{2}\left(\mathbb{R}^{3}\right)}^{2}, \\
C_{3}(t)=C \nu^{-1} r^{-2 a}\|\nabla u\|_{L^{2}\left(\mathbb{R}^{3}\right)}^{4-2 a}\|u\|_{L^{2}\left(\mathbb{R}^{3}\right)}^{2 a} .
\end{gathered}
$$

Нам известно, что положительные функции $C_{1}(t), C_{2}(t)$ и $C_{3}(t)$ интегрируемы по времени. При желании читатель может проверить, что неравенство выше является корректным соотношением между размерными величинами: все члены имеют размерность $[\mathrm{L}]^{6}[\mathrm{~T}]^{-4}$. Теперь получаем

$$
\frac{y^{2}}{1+y} \frac{d y}{d t} \leqslant C_{1}(t)+C_{2}(t)+C_{3}(t)
$$

(что уже не является корректным соотношением с точки зрения размерностей) и после несложного интегрирования мы видим, что у имеет априорную оценку, равномерную по времени. Это доказывает первый критерий.

Для доказательства второго критерия снова воспользуемся представлением $p=\pi(x, r)+\beta(x, r)$, где $r=r(t)$, и найдем оценку для интеграла, содержащего $\pi$, с помощью непосредственного интегрирования по частям и неравенств Гёльдера:

$$
\begin{aligned}
& \left|\int_{\mathbb{R}^{3}}(u \cdot \nabla \pi)\right| u|d x|=\left|\int_{\mathbb{R}^{3}} \pi(u \cdot \nabla|u|) d x\right| \\
& \quad \leqslant \frac{\nu}{2} \int_{\mathbb{R}^{3}}|u||\nabla u|^{2} d x+\frac{C}{\nu}\|u\|_{L^{3}\left(\mathbb{R}^{3}\right)}\|\pi\|_{L^{3}\left(\mathbb{R}^{3}\right)}^{2} .
\end{aligned}
$$

Интеграл, содержащий $\beta$, оценим как в случае первого критерия. Как итог, $y(t)=\|u\|_{L^{3}\left(\mathbb{R}^{3}\right)}$ должно удовлетворять неравенству

$$
y^{2} \frac{d y}{d t} \leqslant C_{4}(t) y+C_{3}(t) y
$$

где по условию $C_{4}(t)=(C / \nu)\|\pi\|_{L^{3}\left(\mathbb{R}^{3}\right)}^{2}$ интегрируемо по времени. Опять мы получаем для $y(t)$ априорную оценку, равномерную по времени. 


\section{6. Приложение}

Здесь мы докажем тождества (5) и (6). Введем полярные координаты

$$
\begin{aligned}
& \xi_{1}=\rho \cos \phi \sin \theta=\rho c S, \\
& \xi_{2}=\rho \sin \phi \sin \theta=\rho s S, \\
& \xi_{3}=\rho \cos \theta=\rho C,
\end{aligned}
$$

где для упрощения обозначений мы полагаем $s=\sin \phi, S=\sin \theta, c=\cos \phi$, $C=\cos \theta$. В случае функций на единичной сфере $\rho=1$, но в общем случае $f(\xi)=f(\rho c S, \rho s S, \rho C)$ и выполняются соотношения

$$
\begin{aligned}
f_{\theta} & =\partial_{\theta} f=\rho\left(c C f_{1}+s C f_{2}-S f_{3}\right), \\
f_{\phi} & =\partial_{\phi} f=\rho\left(-s S f_{1}+c S f_{2}\right), \\
\rho f_{\rho} & =\rho \partial_{\rho} f=\rho\left(c S f_{1}+s S f_{2}+C f_{3}\right),
\end{aligned}
$$

где $\rho \partial_{\rho} f=\xi \cdot \nabla_{\xi} f$, a $\nabla_{\xi} f=\left(f_{1}, f_{2}, f_{3}\right)$. Заметим, что $\rho \partial_{\rho}(\xi /|\xi|)=0$ при $\xi \neq 0$. Имеем

$$
\begin{aligned}
& C f_{\theta}+S \rho f_{\rho}=\rho\left(c f_{1}+s f_{2}\right), \\
& C \rho f_{\rho}-S f_{\theta}=\rho f_{3},
\end{aligned}
$$

так что

$$
\begin{aligned}
& \rho f_{1}=c\left(C f_{\theta}+S \rho f_{\rho}\right)-\frac{s}{S} f_{\phi}, \\
& \rho f_{2}=s\left(C f_{\theta}+S \rho f_{\rho}\right)+\frac{c}{S} f_{\phi}, \\
& \rho f_{3}=C \rho f_{\rho}-S f_{\theta} .
\end{aligned}
$$

Теперь положим $\rho=1$ и обозначим для простоты $D_{\rho}=\rho \partial_{\rho}$. Сначала вычислим

$$
f_{|\xi|=1} \xi_{1} \partial_{1} f(x+r \xi) d S(\xi),
$$

разумеется, воспользовавшись тем, что

$$
d S(\xi)=S d \phi d \theta
$$

Мы получаем равенство

$$
\begin{aligned}
\xi_{1} \partial_{\xi_{1}} f & =c S\left(c\left(C \partial_{\theta}+S D_{\rho}\right)-\frac{s}{S} \partial_{\phi}\right) f \\
& =D_{\rho}\left(\xi_{1}^{2} f\right)+c^{2} S C \partial_{\theta} f-s c \partial_{\phi} f
\end{aligned}
$$

Здесь мы воспользовались тем, что на единичной сфере $\xi=\xi /|\xi|$ и $D_{\rho}(\xi)=0$. Теперь умножим на $S$ и возьмем интеграл, прибегая к интегрированию по частям там, где возможно. Поскольку

$$
-c^{2} \frac{d}{d \theta}\left(S^{2} C\right)=c^{2} S\left(S^{2}-2 C^{2}\right)=c^{2} S\left(3 S^{2}-2\right)
$$


И

$$
S \frac{d}{d \phi}(s c)=2 c^{2} S-S,
$$

коэффициент при $f$ получается сложением:

$$
c^{2} S\left(3 S^{2}-2\right)+2 c^{2} S-S=S\left(3 \xi_{1}^{2}-1\right),
$$

так что

$$
\begin{aligned}
f_{|\xi|=1} \xi_{1} \partial_{\xi_{1}} f d S(\xi) & =f_{|\xi|=1}\left[D_{\rho}\left(\xi_{1}^{2} f\right)+3 \xi_{1}^{2} f-f\right] d S(\xi) \\
& =D_{\rho}\left[\int_{|\xi|=1} \xi_{1}^{2} f d S(\xi)\right]+f_{|\xi|=1}\left(3 \xi_{1}^{2}-1\right) f d S(\xi),
\end{aligned}
$$

что дает нам первое соотношение в (5). Прочие формулы в (5) доказываются аналогично. В самом деле,

$$
\xi_{2} \partial_{\xi_{2}} f=s S\left[s\left(C \partial_{\theta}+S D_{\rho}\right)+\frac{c}{S} \partial_{\theta}\right] f=\left[s^{2} S^{2} D_{\rho}+s^{2} S C \partial_{\theta}+s c \partial_{\phi}\right] f .
$$

Умножая на $S$ и проинтегрировав по частям в членах, содержащих $\partial_{\theta}$ и $\partial_{\phi}$, получим коэффициент при $f$ :

$$
\begin{aligned}
-s^{2} \frac{d}{d \theta}\left(S^{2} C\right)-S \frac{d}{d \phi}(s c) & =s^{2} S\left(3 S^{2}-2\right)+S-2 c^{2} S \\
& =s^{2} S\left(3 S^{2}-2\right)-S+2 s^{2} S=\left(3 \xi_{2}^{2}-1\right) S
\end{aligned}
$$

так что

$$
f_{|\xi|=1} \xi_{2} \partial_{\xi_{2}} f d S(\xi)=D_{\rho}\left[f_{|\xi|=1} \xi_{2}^{2} f d S(\xi)\right]+f_{|\xi|=1}\left(3 \xi_{2}^{2}-1\right) f d S(\xi)
$$

аналогично равенству выше. Третий член равен

$$
\xi_{3} \partial_{3} f=C\left(C D_{\rho}-S \partial_{\theta}\right) f .
$$

Умножая на $S$ и проинтегрировав по частям член, содержащий $\partial_{\theta}$, мы найдем коэффициент при $f$ :

$$
\frac{d}{d \theta}\left(C S^{2}\right)=\left(3 C^{2}-1\right) S=3 \xi_{3}^{2}-1
$$

и тем самым получим последнее соотношение в (5):

$$
f_{|\xi|=1} \xi_{3} \partial_{\xi_{3}} f d S(\xi)=D_{\rho}\left[f_{|\xi|=1} \xi_{3}^{2} f d S(\xi)\right]+f_{|\xi|=1}\left(3 \xi_{3}^{2}-1\right) f d S(\xi) .
$$

Аналогичным образом докажем соотношения (6). Начнем с равенства, отвечающего индексам $(1,3)$ :

$$
\begin{aligned}
\left(\xi_{1} \partial_{\xi_{3}}+\xi_{3} \partial_{\xi_{1}}\right) f & =\left[c S\left(C D_{\rho}-S \partial_{\theta}\right)+C\left(c\left(C \partial_{\theta}+S D_{\rho}\right)-\frac{s}{S} \partial_{\phi}\right)\right] f \\
& =\left[2 c S C D_{\rho}+\left(c C^{2}-c S^{2}\right) \partial_{\theta}-\frac{C s}{S} \partial_{\phi}\right] f
\end{aligned}
$$


Умножив на $S$, взяв интеграл и проинтегрировав по частям, мы найдем коэффициент при $f$ с помощью равенства

$$
-c \frac{d}{d \theta}\left(S\left(1-2 S^{2}\right)\right)+C \frac{d}{d \phi}(s)=-c\left(C-6 S^{2} C\right)+C c=6 c S C S=6 \xi_{1} \xi_{3} S,
$$

так что

$$
\begin{aligned}
f_{|\xi|=1}\left(\xi_{1} \partial_{\xi_{3}}+\xi_{3} \partial_{\xi_{1}}\right) f d S(\xi) & =f_{|\xi|=1}\left[2 \xi_{1} \xi_{3} D_{\rho} f+6 \xi_{1} \xi_{3} f\right] d S(\xi) \\
& =D_{\rho}\left[f_{|\xi|=1} 2 \xi_{1} \xi_{3} f d S(\xi)\right]+f_{|\xi|=1} 6 \xi_{1} \xi_{3} f d S(\xi),
\end{aligned}
$$

что дает соотношение (6) для индексов $(1,3)$. Для индексов $(1,2)$ нам надо вычислить

$$
\begin{aligned}
\left(\xi_{1} \partial_{2}+\xi_{2} \partial_{1}\right) f & =\left[c S\left(s S D_{\rho}+s C \partial_{\theta}+\frac{c}{S} \partial_{\phi}\right)+s S\left(c S D_{\rho}+c C \partial_{\theta}-\frac{s}{S} \partial_{\phi}\right)\right] f \\
& =2 c S s S D_{\rho} f+2 c s S C \partial_{\theta} f+\left(c^{2}-s^{2}\right) \partial_{\phi} f .
\end{aligned}
$$

Умножив на $S$ и проинтегрировав по частям, мы найдем коэффициент $f$, используя равенство

$$
\begin{aligned}
-2 c s \frac{d}{d \theta}\left(S^{2} C\right)-S \frac{d}{d \phi}\left(c^{2}-s^{2}\right) & =2 c s\left(S^{3}-2 S C^{2}\right)+4 S c s \\
& =2 c s\left(S^{3}-2 S+2 S^{3}\right)+4 c s S=6 c s S^{3}=6 \xi_{1} \xi_{2} S .
\end{aligned}
$$

Таким образом, мы получаем

$$
\begin{aligned}
f_{|\xi|=1}\left(\xi_{1} \partial_{\xi_{2}}+\xi_{2} \partial_{\xi_{3}}\right) f d S(\xi) & =f_{|\xi|=1}\left[2 \xi_{1} \xi_{2} D_{\rho} f+6 \xi_{1} \xi_{2} f\right] d S(\xi) \\
& =D_{\rho}\left[f_{|\xi|=1} 2 \xi_{1} \xi_{2} f d S(\xi)\right]+f_{|\xi|=1} 6 \xi_{1} \xi_{2} f d S(\xi)
\end{aligned}
$$

что и дает соотношение $(6)$ для индексов $(1,2)$. Наконец, для индексов $(2,3)$ мы должны вычислить

$$
\begin{aligned}
\left(\xi_{2} \partial_{3}+\xi_{3} \partial_{2}\right) f & =s S\left(C D_{\rho}-S \partial_{\theta}\right) f+C\left(s S D_{\rho}+s C \partial_{\theta}+\frac{c}{S} \partial_{\phi}\right) f \\
& =2 s S C D_{\rho} f+\left(s\left(C^{2}-S^{2}\right) \partial_{\theta}+C \frac{c}{S} \partial_{\phi}\right) f
\end{aligned}
$$

Умножив на $S$ и проинтегрировав по частям, мы найдем коэффициент при $f$, используя равенство

$$
-s \frac{d}{d \theta}\left(S\left(C^{2}-S^{2}\right)\right)-C \frac{d}{d \phi} c=s\left(6 S^{2} C-C\right)+C s=6 s S C S=6 \xi_{2} \xi_{3} S,
$$

что дает нам

$$
\begin{aligned}
f_{|\xi|=1}\left(\xi_{2} \partial_{\xi_{3}}+\xi_{3} \partial_{\xi_{2}}\right) f d S(\xi) & =f_{|\xi|=1}\left[2 \xi_{2} \xi_{3} D_{\rho} f+6 \xi_{2} \xi_{3} f\right] d S(\xi) \\
& =D_{\rho}\left[f_{|\xi|=1} 2 \xi_{2} \xi_{3} f d S(\xi)\right]+f_{|\xi|=1} 6 \xi_{2} \xi_{3} f d S(\xi),
\end{aligned}
$$

т. е. соотношение (6) для индексов $(2,3)$. 


\section{Список литературы}

[1] L. C. Berselli, G.P. Galdi, "Regularity criteria involving the pressure for the weak solutions to the Navier-Stokes equations", Proc. Amer. Math. Soc., 130:12 (2002), 3585-3595.

[2] P. Constantin, "An Eulerian-Lagrangian approach to the Navier-Stokes equations", Comm. Math. Phys., 216:3 (2001), 663-686.

[3] L. Escauriaza, G. Seregin, V. Šverák, "Backward uniqueness for parabolic equations", Arch. Ration. Mech. Anal., 169:2 (2003), 147-157.

[4] C. Foiaş, C. Guillopé, R. Temam, "New a priori estimates for Navier-Stokes equations in dimension 3", Comm. Partial Differential Equations, 6:3 (1981), 329-359.

[5] P. Isett, Regularity in time along the coarse scale flow for the incompressible Euler equations, 2013 (v3 - 2014), 48 pp., arXiv: 1307.0565.

[6] G. Seregin, V. Šverák, "The Navier-Stokes equations and backward uniqueness", Nonlinear problems in mathematical physics abd related topics. II, Int. Math. Ser. (N. Y.), 2, Kluwer/Plenum, New York, 2002, 353-366.

[7] G. Seregin, V. Šverák, "Navier-Stokes equations with lower bounds on the pressure", Arch. Ration. Mech. Anal., 163:1 (2002), 65-86.

[8] T. Tao, "Localisation and compactness properties of the Navier-Stokes global regularity problem", Anal. PDE, 6:1 (2013), 25-107; 2012, 95 pp., arXiv: 1108.1165v4.

[9] A. Vasseur, "Higher derivatives estimate for the 3D Navier-Stokes equation", Ann. Inst. H. Poincaré Anal. Non Linéaire, 27:5 (2010), 1189-1204.

Питер Константин

(Peter Constantin)

Princeton University, Princeton, USA

E-mail: const@math.princeton.edu
Поступила в редакцию

27.10 .2013 